\title{
An Energy-aware Greedy Perimeter Stateless Routing Protocol for Mobile Ad hoc Networks
}

\author{
Natarajan Meghanathan \\ Jackson State University \\ P. O. Box 18839, 1400 J. Lynch Street \\ Jackson, MS 39217, USA
}

\begin{abstract}
The high-level contribution of this paper is an energy-aware version of the well-known position-based Greedy Perimeter Stateless Routing (GPSR) protocol for mobile ad hoc networks (MANETs). In the proposed energy-aware GPSR protocol, referred to as E-GPSR, we optimize the greedy forwarding mode as follows: a forwarding node first determines a candidate set of neighbor nodes - the nodes that lie closer to the destination than itself. The weight of each such candidate neighbor node is then computed to be the sum of the fraction of the initial energy currently available at the neighbor node and the progress (i.e., the fraction of the distance covered between the forwarding node and the destination) obtained with the selection of the neighbor node. The candidate neighbor node that has the largest weight value is the chosen next hop node to receive the data packet. This procedure is repeated at every hop where greedy forwarding is possible. In case, greedy forwarding is not possible, similar to GPSR, E-GPSR switches to perimeter forwarding. With E-GPSR, the fairness of node usage considerably improves compared to GPSR. Simulation results illustrate that the time of first node failure, due to the exhaustion of battery charge, increases significantly (as large as by 55\%) with the use of E-GPSR, whereas the hop count increases only as large as by $7 \%$.
\end{abstract}

\section{General Terms}

Algorithms

\section{Keywords}

Greedy Routing, Energy-awareness, Lifetime, Mobile Ad hoc Networks, Simulation

\section{INTRODUCTION}

A mobile ad hoc network (MANET) is a dynamic distributed system of wireless nodes that move independently of each other. The operating transmission range of the nodes is limited and as a result, MANET routes are often multi-hop in nature. Any node in a MANET can become a source or destination, and each node can function as a router, forwarding data for its peers. MANET routing protocols can be classified into topology-based and position-based protocols. Topology-based protocols are either proactive or reactive in nature. Proactive routing protocols determine and maintain routes between any pair of nodes irrespective of their requirement. The reactive on-demand routing protocols determine a route only when required. As the network topology changes dynamically, reactive on-demand routing has been preferred over proactive routing [1][2].

Position-based routing protocols do not conduct on-demand route discovery to learn and maintain routes. Instead, forwarding decisions are taken independently for each data packet at every forwarding node (including the source) depending on the position of the forwarding node, the intermediate nodes and the destination. Normally, the source includes its estimated location information of the destination in every data packet. The positionbased routing protocols are mostly designed to choose the intermediate forwarding nodes that lie on the shortest path or close to the shortest path from the source to the destination. Greedy Perimeter Stateless Routing (GPSR) [3] is a well-known and most commonly used position-based routing protocol for MANETs. GPSR works as follows: The source periodically uses a location service scheme (e.g., [4]) to learn about the latest location information of the destination and includes it in the header of every data packet. If the destination is not directly reachable, the source node forwards the data packet to the neighbor node that lies closest to the destination. Such a greedy procedure of forwarding the data packets is also repeated at the intermediate nodes. In case, a forwarding node could not find a neighbor that lies closer to the destination than itself, the node switches to perimeter forwarding. With perimeter forwarding, the data packet is forwarded to the first neighbor node that is come across, when the line connecting the forwarding node and the destination of the data packet is rotated in the anti-clockwise direction. The location of the forwarding node in which greedy forwarding failed (and perimeter forwarding began to be used) is recorded in the data packet. We switch back to greedy forwarding when the data packet reaches a forwarding node which can find a neighbor node that is away from the destination node by a distance smaller than the distance between the destination node and the node at which perimeter forwarding began.

During both greedy forwarding and perimeter forwarding, the energy available at the chosen neighbor node to forward the data packet is not considered. In networks of moderate and high density, greedy forwarding happens to be used more than $98 \%$ of the time and the need for perimeter forwarding is highly unlikely. This motivated us to optimize the greedy forwarding phase of GPSR by considering the energy available at the neighbor nodes of a forwarding node before deciding the next hop node for transmitting the data packet. Accordingly, we propose an energyaware version of GPSR (E-GPSR) that operates as follows: a forwarding node first determines a candidate set of neighbor nodes - the nodes that lie closer to the destination than itself. The weight of each such candidate neighbor node is then computed to be the sum of the fraction of the initial energy currently available at the neighbor node and the progress (i.e., the fraction of the distance covered between the forwarding node and the destination) obtained with the selection of the neighbor node. The candidate neighbor node that has the largest weight value is the chosen next hop node to receive the data packet. This procedure is repeated at every hop where greedy forwarding is possible. In 
case, greedy forwarding is not possible, similar to GPSR, E-GPSR switches to perimeter forwarding.

Both GPSR and E-GPSR require each node periodically (for every one second, in this paper) broadcast a beacon containing its latest location information to its neighbors. A beacon message broadcast by a node includes the node ID, the current X and Y coordinate location of the node (for both GPSR and E-GPSR) and the fraction of the initial energy currently available at the node (for E-GPSR alone). The size of the node ID field is 2 bytes and the size of each other field in the beacon message is 8 bytes. Hence, the total size of a beacon message is 20 bytes and 28 bytes with GPSR and E-GPSR respectively.

We hypothesize that compared to GPSR, with the use of E-GPSR, the unfairness in node usage would relatively reduce and the time of first node failure due to the exhaustion of the available battery charge would relatively increase. The performance of E-GPSR vis-à-vis GPSR has been evaluated through extensive simulations and we did observe our hypothesis to be correct. The relative increase in the hop count per source-destination path, as a result of considering the energy available at the neighbor nodes in addition to the distance covered, has been very minimal, as large as only by $7 \%$. Thus, E-GPSR is a valuable addition to the MANET literature on position-based routing protocols.

Note that the effectiveness of the position-based routing protocols depends on the accuracy of the destination location information included in the header of the data packets, method adopted for disseminating the location information and the method adopted to learn the latest location information of the destination node. Like in all literary works that propose and evaluate a position-based routing protocol, we assume the availability of a location service that is made use of by the source node to know the exact location information of the destination node and included in every data packet. In future work, we will evaluate the impact of the location-service schemes on the performance of GPSR vis-à-vis E-GPSR. The rest of the paper is organized as follows: In Section 2, we explain the greedy forwarding algorithm used in GPSR. Section 3 describes the proposed greedy forwarding algorithm for E-GPSR to select the next hop neighbor for data transmission at a forwarding node. Section 4 describes the simulation environment and presents the simulation results. Section 5 discusses related work. Section 6 concludes the paper and lists the future work.

\section{GREEDY FORWARDING ALGORITHM USED IN GPSR}

Let $\left(X_{D}, Y_{D}\right)$ and $\left(X_{F}, Y_{F}\right)$ respectively denote the locations of the destination node $D$ and the forwarding node $F$ that has the data packet addressed to the destination node $D$. Figure 1 illustrates the pseudo code for the greedy algorithm used at a forwarding node in the traditional GPSR. The forwarding node $F$ computes the distance between itself and the destination node $D$ as well as the distance between each of its neighbor nodes and $D$. If a neighboring node $I$ of the forwarding node $F$ is relatively closer to $D$, then the progress (i.e. fraction of the distance covered) with the potential selection of $I$ as the next hop node would be the difference in the distance between $F$ and $D$ and the distance between $I$ and $D$ divided by the distance between $F$ and $D$. Among such neighbor nodes, the neighbor node that has the maximum value for the progress is the node that lies closest to the destination and is chosen by $F$ as the next hop to forward the data packet. If the forwarding node $F$ could not find a neighbor node that lies closer to the destination than itself, then the node switches to perimeter forwarding.

Input: Forwarding Node $F$, Destination $D$, Neighbor-List $(F)$

Auxiliary Variables: Progress $(F, I)$ where $I \in$ Neighbor-List $(F)$ Maximum-Progress

Output: Next-Hop-Node // if Greedy forwarding is successful

NULL // if Greedy forwarding is not successful and perimeter forwarding is needed

Initialization: Next-Hop-Node $=$ NULL Maximum-Progress $\leftarrow 0.0$

Begin GPSR Greedy Forwarding Algorithm

Distance $_{F-D}=\sqrt{\left(X_{F}-X_{D}\right)^{2}+\left(Y_{F}-Y_{D}\right)^{2}}$

for every neighbor node $I \in$ Neighbor-List $(F)$ do

$$
\begin{aligned}
& \text { Distance }_{I-D}=\sqrt{\left(X_{I}-X_{D}\right)^{2}+\left(Y_{I}-Y_{D}\right)^{2}} \\
& \text { if }\left(\text { Distance }_{I-D}<\text { Distance }_{F-D}\right) \text { then } \\
& \operatorname{Pr} \operatorname{ogress}(F, I)=\frac{{\text { Dis } \tan c e_{F-D}-\text { Dis }_{\tan } e_{I-D}}_{\text {Dis } \tan c e_{F-D}}}{\text { P }_{F-D}} \\
& \text { if (Maximum-Progress }<\text { Progress }(F, I) \text { ) then } \\
& \text { Maximum-Progress }=\text { Progress }(F, I) \\
& \text { Next-Hop-Node } \leftarrow I \\
& \text { end if }
\end{aligned}
$$
if (Maximum-Progress $>0.0$ ) then
return Next-Hop-Node // Greedy forwarding is successful else
return NULL // Greedy forwarding is not successful and $/ /$ perimeter forwarding is needed

end if

End GPSR Greedy Forwarding Algorithm

Figure 1. Greedy Forwarding Algorithm used in GPSR

\section{GREEDY FORWARDING ALGORITHM USED IN E-GPSR}

Let $\left(X_{D}, Y_{D}\right)$ and $\left(X_{F}, Y_{F}\right)$ respectively denote the locations of the destination node $D$ and the forwarding node $F$ that has the data packet addressed to the destination node $D$. Figure 2 illustrates the pseudo code for the proposed greedy algorithm used at a forwarding node in E-GPSR. We first form a candidate set of neighboring nodes, Candidate-Neighbor-List $(F)$, which is a subset of the Neighbor-List $(F)$. For every neighbor $I$ $\in$ Neighbor-List $(F), I \in$ Candidate-Neighbor-List $(F)$, if and only if, the distance between the neighbor node $I$ and the destination node $D$ is less than the distance between the forwarding node $F$ and $D$. For every neighbor node $I \in$ Candidate-Neighbor-List $(F)$, we then compute a Weight $(I)$, defined as the sum of the (a) fraction of the initial energy currently available at $I$, referred to as ResidualEnergy (I), and the 
(b) fraction of the distance covered with the potential selection of $I$, referred to as Progress $(F, I)$, which would be the difference in the distance between $F$ and $D$ and the distance between $I$ and $D$ divided by the distance between $F$ and $D$. Among such neighbor nodes, the neighbor node that has the maximum Weight value is chosen by $F$ as the next hop node to forward the data packet. If the forwarding node $F$ could not find a neighbor node that lies closer to the destination than itself, the Candidate-Neighbor-List is empty and the node switches to perimeter forwarding.

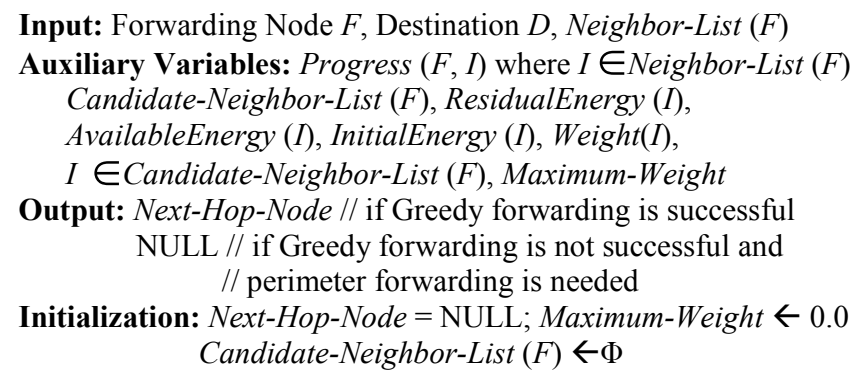

Begin E-GPSR Greedy Forwarding Algorithm

Distance $_{F-D}=\sqrt{\left(X_{F}-X_{D}\right)^{2}+\left(Y_{F}-Y_{D}\right)^{2}}$

for every neighbor node $I \in$ Neighbor-List $(F)$ do

\author{
Distance $_{I-D}=\sqrt{\left(X_{F}-X_{D}\right)^{2}+\left(Y_{F}-Y_{D}\right)^{2}}$ \\ if (Distance $_{I-D}<$ Distance $\left._{F-D}\right)$ then \\ Candidate-Neighbor-List $(F) \leftarrow$ Candidate-Neighbor-List $(F) \mathrm{U}\{I\}$ \\ end if \\ end for
}

for every neighbor node $I \in$ Candidate-Neighbor-List $(F)$ do

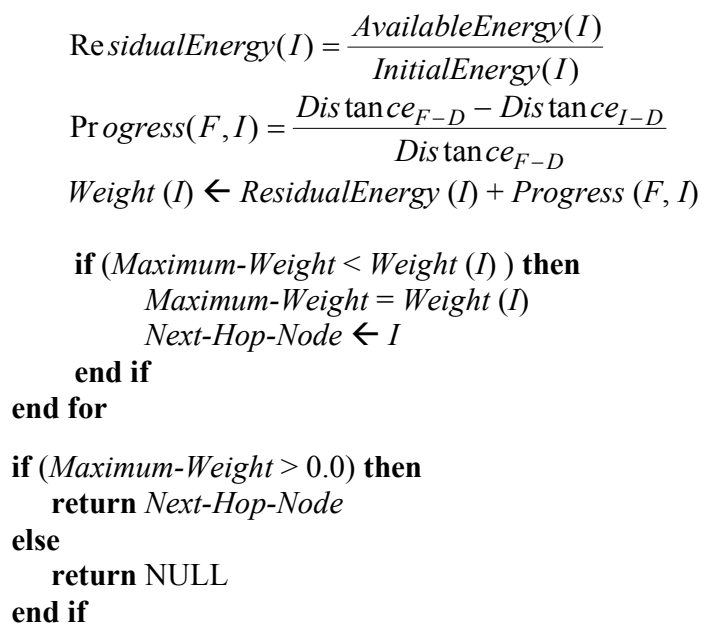

End E-GPSR Greedy Forwarding Algorithm

Figure 2. Greedy Forwarding Algorithm used in E-GPSR With the above described energy-aware approach, the neighbor node that lies farthest away from the forwarding node need not be always selected as the next hop node and a neighbor that has a relatively larger available residual energy and located relatively closer to the destination, compared to the forwarding node, could be chosen as the next hop. This could significantly maximize the time of first node failure especially in static and quasi-static ad hoc networks, where there are no significant neighborhood changes. The energy-aware neighbor selection of E-GPSR has the potential to very well balance the forwarding load among all the neighbor nodes rather than always using the neighbor node that lies farthest away from the forwarding node and closest to the destination. Note that the percentage of time instants a node gets into perimeter forwarding is the same in the case of both GPSR and E-GPSR. In other words, if greedy forwarding is successful in GPSR, greedy forwarding would also be successful in E-GPSR and vice-versa.

\section{SIMULATIONS}

We use ns-2 (version 2.28) [5] as the simulator for our study. We implemented the GPSR and E-GPSR protocols in ns-2. The network dimension used is a $1000 \mathrm{~m} \times 1000 \mathrm{~m}$ square network. The transmission range of each node is assumed to be $250 \mathrm{~m}$. The number of nodes used is 50 and 100 nodes representing networks of moderate (on the average 10 neighbors per node) and high density (on the average 20 neighbors per node) respectively where greedy forwarding is predominantly more common over perimeter forwarding. We chose such network density conditions so that the impact of the energy-aware greedy forwarding technique on GPSR can be exploited to the maximum. The average network connectivity at these density values is more than $99 \%$ and greedy forwarding is used for at least $98 \%$ of the packets sent from each source node. Initially, nodes are uniform-randomly distributed in the network. We assume the availability of an off-line location service scheme through which the source node can learn the exact location of the destination node at the time of sending a data packet. The Medium Access Control (MAC) layer model used is the IEEE 802.11 [6] model. Link bandwidth is $2 \mathrm{Mbps}$. The interface queue is FIFO-based with a queue size of 100.

Traffic sources are constant bit rate (CBR). The number of source-destination $(s-d)$ sessions used is 15 (low traffic load) and 30 (high traffic load). The starting timings of these $s-d$ sessions are uniform-randomly distributed between 1 to 10 seconds. The sessions continue until the end of the simulation time, which is 1000 seconds. Data packets are 512 bytes in size and the packet sending rate is 4 data packets/second. For each node, we made sure that the node does not end up as a source for more than two sessions and/ or not as a destination for more than two sessions.

The node mobility model used is the Random Waypoint model [7], a widely used mobility model in MANET simulation studies. According to this model, each node starts moving from an arbitrary location to a randomly selected destination location at a speed uniformly distributed in the range $\left[v_{\min }, \ldots, v_{\max }\right]$. Once the destination is reached, the node may stop there for a certain time called the pause time and then continue to move by choosing a different target location and a different velocity. In this paper, we set $v_{\min }=0$, and each node chooses a speed uniformly distributed between 0 and $v_{\max }$. The $v_{\max }$ values used are $5 \mathrm{~m} / \mathrm{s}, 25 \mathrm{~m} / \mathrm{s}$ and 50 $\mathrm{m} / \mathrm{s}$ representing scenarios of low, moderate and high node mobility respectively. Pause time is 0 seconds.

\subsection{Performance Metrics}

We measure the following performance metrics for the simulation conditions listed above. Each of the data points in the 
performance figures illustrated in figures 3 through 5 are average values obtained after running simulations of ten different node mobility profiles for a given condition of node mobility (i.e., $v_{\max }$ ), offered traffic load and network density.

(i) Time of First Node Failure: The time of first node failure due to the exhaustion of battery charge during the simulation of a particular routing protocol is a measure of the node lifetime.

(ii) Hop Count per Source-Destination $(s-d)$ Path: The hop count per $s-d$ path is the average of the number of hops in the routes of an $s-d$ session, time-averaged after considering all the $s-d$ sessions. For example, if a routing protocol uses paths $\mathrm{P} 1$ and $\mathrm{P} 2$ of hop counts 3 and 5 for time 10 seconds and 5 seconds respectively, then the time-averaged hop count for the total time of 15 seconds is $(3 * 10+5 * 5) / 15=3.67$ seconds and not simply 4 seconds.

(iii) Unfairness Index: The unfairness index is computed as the standard deviation of the energy consumed per node, which is the square root of the average of the squares of the difference between the energy consumed at each node and the average energy consumed per node. Ideally, the value of this metric should be zero to indicate that all nodes have been used fairly and no node is overused.

\subsection{Time of First Node Failure}

E-GPSR yields significantly larger values for the time of first node failure, compared to GPSR. This can be attributed to the equal importance given to the residual energy available at a neighbor node as well as the progress made on the distance covered, from the forwarding node to the destination node, through the neighbor node. E-GPSR fairly balances both of these measures - does not excessively use neighbor nodes that have the maximum progress as the forwarding nodes and at the same time does not significantly increase the hop count by always picking the neighbor node with the maximum residual energy.

For a given network density and offered traffic load, the difference in the times of first node failure between E-GPSR and GPSR mostly decreases with increase in the maximum node velocity. This is due to the dynamic changes in the neighborhood of a forwarding node with increase in node mobility and a neighbor node need not always lie on the minimum hop path of maximum progress from the source to the destination, as in the case of GPSR. The improvement in the time of first node failure for E-GPSR vis-à-vis GPSR is $15 \%-55 \%$ in scenarios of low node mobility and $15 \%-26 \%$ in scenarios of high node mobility.

For a given network density and node mobility, the difference in the times of first node failure between E-GPSR and GPSR increases with increase in the offered traffic load. E-GPSR takes into consideration the available residual energy at the neighbor nodes of a forwarding node before deciding on the next hop node. This helps to significantly extend the lifetime of the nodes in heavy traffic scenarios - rather than always choosing the node with the maximum progress (i.e. distance covered) as in GPSR, EGPSR helps to extend the lifetime of nodes that are close to be completely depleted of their battery charge. The percentage improvement in the time of first node failure for E-GPSR vis-àvis GPSR is $15 \%-55 \%$ in scenarios of high offered traffic load and $15 \%-25 \%$ in scenarios of low offered traffic load.

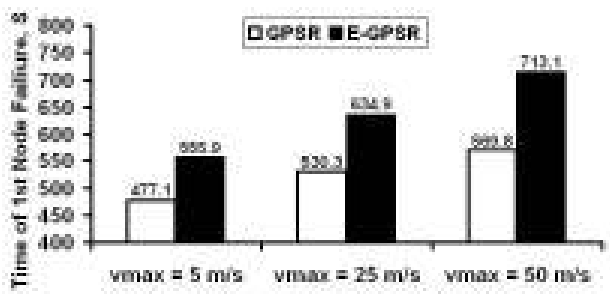

Figure 3.1. 50 Nodes and $15 s-d$ Pairs

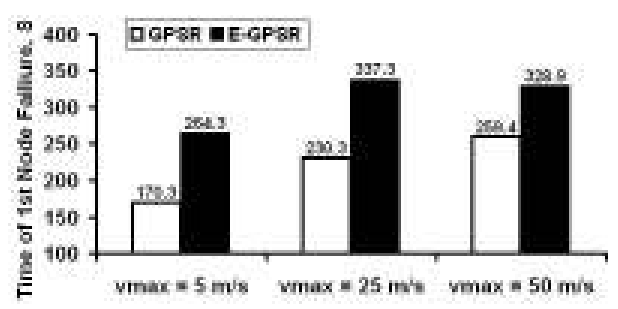

Figure 3.2. 50 Nodes and $30 s$ - $d$ Pairs

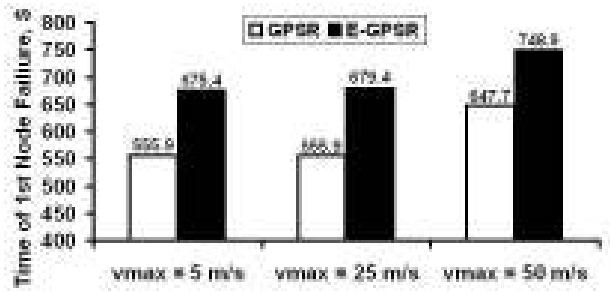

Figure 3.3. 100 Nodes and $15 s-d$ Pairs

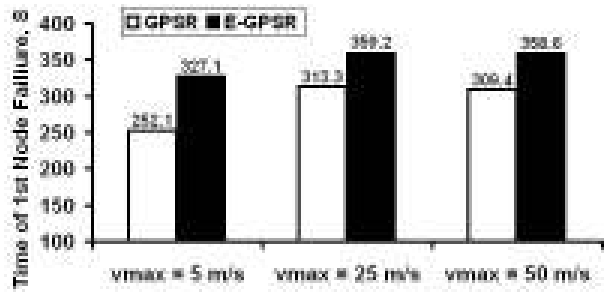

Figure 3.4. 100 Nodes and $30 s-d$ Pairs

Figure 3. Time of First Node Failure (GPSR vs. E-GPSR)

We notice that for a given offered traffic load and node mobility, for both GPSR and E-GPSR, the absolute values of the time of first node failure increases with increase in network density. This could be attributed to the availability of a larger number of nodes in the neighborhood as candidate next hop nodes for the greedy forwarding algorithm. The forwarding load could very well be rotated among these neighbor nodes over time.

For a given condition of network density and offered traffic load, for both GPSR and E-GPSR, we notice that the time of first node failure increases with increase in the maximum node velocity. This can be attributed to the fast dynamic changes in the neighborhood of a forwarding node with increase in the maximum node velocity and a neighbor node is highly unlikely to be used as the next hop forwarding node for all the time. For a given node mobility and network density, for both GPSR and E-GPSR, the time of first node failure decreases with increase in the offered traffic load. This is more obvious as the route forwarding load of a node increases with increase in the number of $s-d$ sessions. 


\subsection{Hop Count per Source-Destination (s-d) Path}

With E-GPSR giving equal weights to the progress obtained and the residual energy available at the neighbor nodes of a forwarding node, the hop count of the $s-d$ paths traversed by the data packets need not be the minimum and could be higher than that incurred with GPSR. But, we notice that the increase in the hop count is not significantly high. The maximum increase in the hop count per $s-d$ path is from 3\% (at high node mobility) to $7 \%$ (at low node mobility). The relatively lower increase in the hop count per $s$ - $d$ path at high node mobility scenarios could be attributed to the dynamically changing network topology and the energy reserves at all the nodes are relatively used more fairly. This is vindicated with the increase in the time of first node failure (Figure 3) and decrease in the Unfairness Index (Figure 5) with increase in the maximum node mobility, for a given network density and offered traffic load.

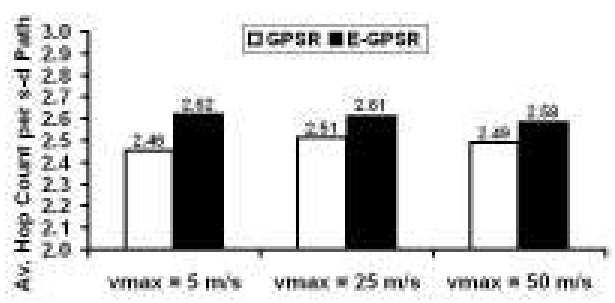

Figure 4.1. 50 Nodes and $15 s-d$ Pairs

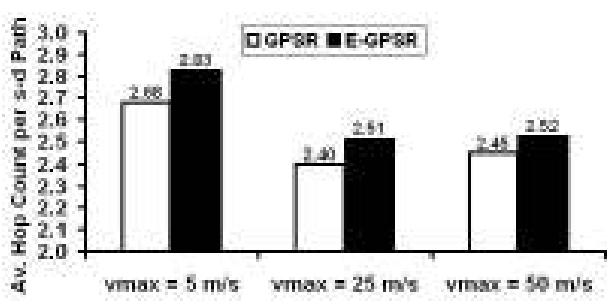

Figure 4.2. 50 Nodes and $30 s$ - $d$ Pairs

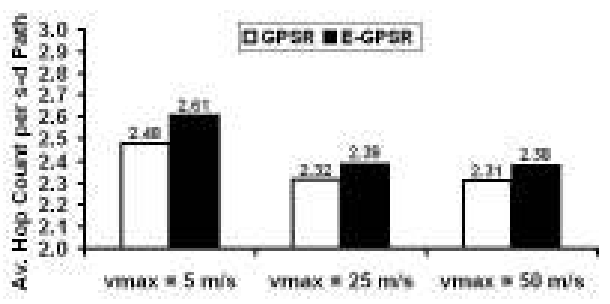

Figure 4.3. 100 Nodes and $15 s-d$ Pairs

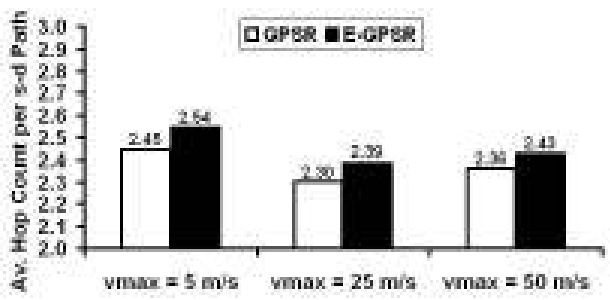

Figure 4.4. 100 Nodes and $30 s$ - $d$ Pairs

Figure 4. Hop Count per Source-Destination Path (GPSR vs. E-GPSR)
We also noticed in our simulations that the slightly larger hop count in E-GPSR did not significantly affect the energy consumed per data packet and the increase in the energy consumed per data packet for E-GPSR vis-à-vis GPSR is only at most $4 \%$. Thus, the net energy consumed in the network due to E-GPSR is almost the same as that of GPSR and there is no significantly high difference. This is one of the key contributing factors to a larger time of first node failure obtained with E-GPSR.

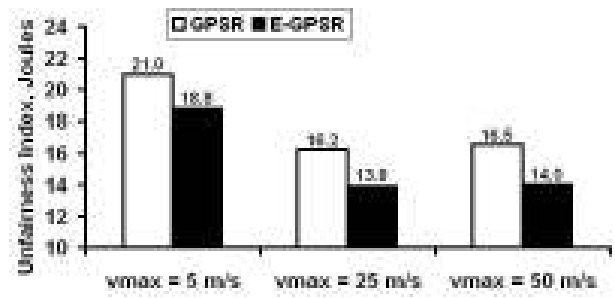

Figure 5.1. 50 Nodes and $15 s$ - $d$ Pairs

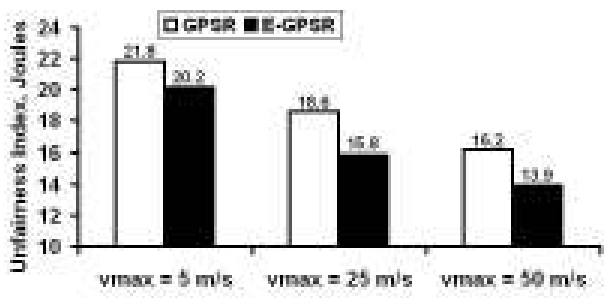

Figure 5.2. 50 Nodes and $30 s-d$ Pairs

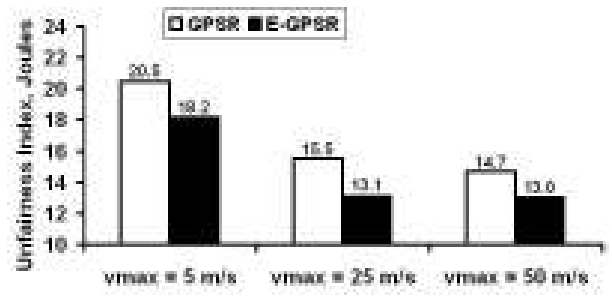

Figure 5.3. 100 Nodes and $15 s-d$ Pairs

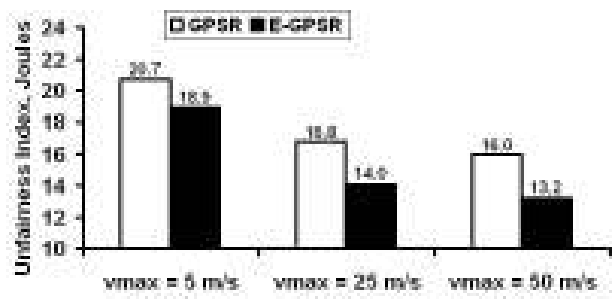

Figure 5.4. 100 Nodes and $30 s-d$ Pairs

Figure 5. Average Unfairness Index (GPSR vs. E-GPSR)

\subsection{Unfairness Index}

The Unfairness Index of E-GPSR is lower than that of GPSR for all the simulation scenarios - the Unfairness Index of E-GPSR is $9 \%-18 \%$ lower than that of GPSR. This illustrates that the EGPSR is able to more fairly utilize the energy reserves at the nodes by giving equal consideration to the progress made from the forwarding node to the destination through a neighbor node as well as to the residual energy available at the neighbor node. The relatively higher fairness in node usage combined with an 
insignificant increase in the hop count and energy consumed per data packet contribute to the significant increase in the time of first node failure for E-GPSR vis-à-vis GPSR.

For a given network density and offered traffic load, the fairness of node usage for both GPSR and E-GPSR increases with increase in node mobility. This could be observed through a decrease in the Unfairness Index for both GPSR and E-GPSR, with increase in the maximum node velocity. For a given offered data traffic load and node mobility, the Unfairness Index for both GPSR and E-GPSR slightly decreases with increase in network density. This could be attributed to the availability of a larger number of nodes (to be the next hop nodes) in the neighborhood of a forwarding node with increase in network density and the route forwarding load can be shared among these nodes.

For a given node mobility and network density, the Unfairness Index of a routing protocol increases with increase in the offered data traffic load. This is more apparent in the case of GPSR as the protocol gives $100 \%$ importance to the maximum progress made at a forwarding node without considering the energy reserves at the candidate neighbor nodes. As a result, any node is highly likely to be chosen as the next hop node for more than one sourcedestination $(s-d)$ session. On the other hand, in the case of EGPSR, the increase in the Unfairness Index with increase in the offered data traffic load is not significantly high - the slight increase could be just attributed to the increase in the number of $s$ $d$ sessions from 15 to 30 . In scenarios of high node mobility, EGPSR actually manages to incur a lower Unfairness Index in high density networks compared to low density networks.

\section{RELATED WORK}

To the best of our knowledge, E-GPSR is the first energy-aware greedy routing protocol proposed for mobile ad hoc networks. The Energy-Aware Greedy Routing (EAGR) scheme proposed in [8] has been tested only for static wireless ad hoc networks. The Geographic and Energy Aware Routing (GEAR) algorithm proposed in [9] for wireless sensor networks works as follows: The entire ad hoc network is divided into grids. If an intermediate node lies within the same grid as that of the destination node, the packet is flooded to the entire grid; otherwise, the packet is forwarded to the best next hop node with the largest weighted cost (of the distance to the destination and the energy available).

\section{CONCLUSIONS AND FUTURE WORK}

We have proposed an energy-aware greedy forwarding algorithm for GPSR, referred to as E-GPSR. E-GPSR considers both the progress made towards the destination as well as the residual energy available at the neighboring nodes of a forwarding node before choosing the next hop towards the destination. Simulation results illustrate that E-GPSR can extend node lifetime by incurring a relatively longer time for the first node failure due to exhaustion of battery charge. The time of first node failure for EGPSR could be as large as 55\% more (compared to GPSR) for scenarios of low node mobility and high offered data traffic load. As node mobility increases, the relative difference between GPSR and E-GPSR with respect to the time of first node failure decreases. Nevertheless, E-GPSR yields at least $15 \%$ larger node lifetime compared to GPSR over all the scenarios considered. The tradeoff is a slightly larger hop count; but the relative increase in hop count per E-GPSR $s$ - $d$ paths vis-à-vis GPSR $s$ - $d$ paths is only at most $7 \%$. This does not result in any significant increase in the energy consumed per data packet and energy consumed per node - contributing to the success of E-GPSR in significantly increasing the node lifetime. E-GPSR attempts to fairly utilize the nodes by taking into consideration the residual energy available at the nodes before deciding the next hop node. As a result, a single node that always lies on the path of maximum progress need not be repeatedly chosen, which may be the case with GPSR, especially in networks of low node mobility and low or moderate network density. E-GPSR fairly rotates the data forwarding load to all the nodes in the neighborhood, thus incurring a relatively lower Unfairness Index compared to GPSR. Thus, E-GPSR is a valuable addition to the literature of position-based MANET routing protocols. In future, we will study the performance GPSR and E-GPSR under different MANET mobility models as well as under different models for unidirectional links. We will also study the performance of GPSR and E-GPSR under different location service schemes and consider the impact of the location update overhead as well as the inaccuracy in location prediction and estimation on the performance of these two routing protocols.

\section{REFERENCES}

[1] J. Broch, D. A. Maltz, D. B. Johnson, Y. C. Hu, J. Jetcheva, "A Performance Comparison of Multi-hop Wireless Ad Hoc Network Routing Protocols," Proceedings of the $4^{\text {th }} A C M$ Annual International Conference on Mobile Computing and Networking, pp.85-97, Dallas, TX, USA, October 1998.

[2] P. Johansson, T. Larsson, N. Hedman, B. Mielczarek and M. Degermark, "Scenario-based Performance Analysis of Routing Protocols for Mobile Ad hoc Networks," Proceedings of the $5^{\text {th }}$ ACM Annual International Conference on Mobile Computing and Networking, pp. 195206, Seattle, WA, USA, August 1999.

[3] B. Karp and H. T. Kung, "GPSR: Greedy Perimeter Stateless Routing for Wireless Networks," Proceedings of the $6^{\text {th }}$ Annual International Conference on Mobile Computing and Networking, pp. 243-254, Boston, MA, USA, August 2000.

[4] W. Keiss, H. Fuessler and J. Widmer, "Hierarchical Location Service for Mobile Ad hoc Networks," ACM SIGMOBILE Mobile Computing and Communications Review, vol. 8, no. 4, pp. 47-58, 2004.

[5] K. Fall and K. Varadhan, "ns notes and documentation," The VINT Project at LBL, Xerox PARC, UCB, and USC/ISI, http://www.isi.edu/nsnam/ns, August 2001.

[6] G. Bianchi, "Performance Analysis of the IEEE 802.11 Distributed Coordination Function," IEEE Journal of Selected Areas in Communication, vol. 18, no. 3, pp. 535547, March 2000.

[7] C. Bettstetter, H. Hartenstein and X. Perez-Costa, "Stochastic Properties of the Random-Way Point Mobility Model," Wireless Networks, vol. 10, no. 5, pp. 555-567, September 2004.

[8] S. Sharma, H. M. Gupta and S. Dharmaraja, "EAGR: Energy Aware Greedy Routing Scheme for Wireless Ad hoc Networks," Proceedings of the SPECTS Conference, pp. 122-129, June 2008.

[9] Y. Yu, R. Govindan and D. Estrin, "Geographical and Energy Aware Routing: A Recursive Data Dissemination Protocol for Wireless Sensor Networks," UCLA Technical Report, UCLA/CSD-TR-01-0023, May 2001. 